\title{
Electronics development for Cherenkov particle identification detectors for PANDA at FAIR
}

\author{
Matteo Cardinali ${ }^{* a b}$, Maria Isabel Ferretti Bondy ${ }^{b}$, Oliver Corell ${ }^{b}$, Werner Lauth ${ }^{b}$, \\ Björn Sören Schlimme ${ }^{b}$, Michaela Thiel ${ }^{b}$, Cahit Ugur ${ }^{c}$ and Concettina Sfienti ${ }^{b}$. \\ ${ }^{a}$ Helmholtz-Institut Mainz, \\ Johann-Joachim-Becher-Weg 36, 55128 Mainz, Germany \\ ${ }^{b}$ Institut für Kernphysik, Johannes Gutenberg-Universität Mainz, \\ Johann-Joachim-Becher-Weg 45, 55128 Mainz, Germany \\ ${ }^{c}$ GSI Helmholtzzentrum für Schwerionenforschung $\mathrm{GmbH}$ \\ Planckstraße 1, 64291 Darmstadt, Germany \\ E-mail: cardinal@kph.uni-mainz.de, ferretti@kph.uni-mainz.de, \\ corell@kph.uni-mainz.de, lauth@kph.uni-mainz.de, \\ schlimmeakph.uni-mainz.de, thielakph.uni-mainz.de, c.uguregsi.de, \\ sfienti@kph.uni-mainz.de
}

Particle identification is of key importance for the $\overline{\mathrm{P}} \mathrm{ANDA}$ detector at the future facility FAIR. A Detector of Internally Reflected Cherenkov light (DIRC) will allow the separation of charged pions and kaons up to $3.5 \mathrm{GeV} / \mathrm{c}$ in the barrel region. Fast signals with high gain are required due to the small number of Cherenkov photons per track and the expected high count rate. MicroChannel Plate (MCP) photomultipliers have been chosen as photosensor, allowing the operation inside a 1 T magnetic field. Dedicated Front-End Electronics (FEE) has been developed in Mainz: the modular system consists of a pre-amplifier board (gain 8) and a NINO ASIC chip board with discriminator function. The readout system was completed by a newly developed TDC Readout Board (TRBv3). The characterization of this new FEE has been performed in the laboratory followed by an in-beam test at MAMI (Mainzer Microtron) of the complete setup. The promising results of these R\&D studies as the future developments will be discussed.

International Winter Meeting on Nuclear Physics,

21-25 January 2013

Bormio, Italy

${ }^{*}$ Speaker. 


\section{Introduction}

The international Facility for Antiproton and Ion Research (FAIR) is currently under construction at the GSI Helmholtzzentrum für Schwerionenforschung in Darmstadt, Germany. The accelerators will provide primary proton and ion beams as well as secondary beams of antiprotons and radioactive ions to several experiments, with high energy, quality and unprecedented intensity. The High Energy Storage Ring (HESR) will accumulate antiprotons with a momentum range of 1.5 to $15 \mathrm{GeV} / \mathrm{c}$ and a momentum resolution of $2 \times 10^{-5}$ due to stochastic and electron cooling. At the interaction point of the $\overline{\mathrm{P}} \mathrm{ANDA}^{1}$ detector, shown in fig. 1, the antiproton beam will collide with a fixed target, allowing to reach an average luminosity of $2 \times 10^{32} \mathrm{~cm}^{-2} \mathrm{~s}^{-1}$ (for $\mathrm{H}_{2}$ target). The interaction rate will be $20 \mathrm{MHz}$ on average with peaks up to $50 \mathrm{MHz}$.

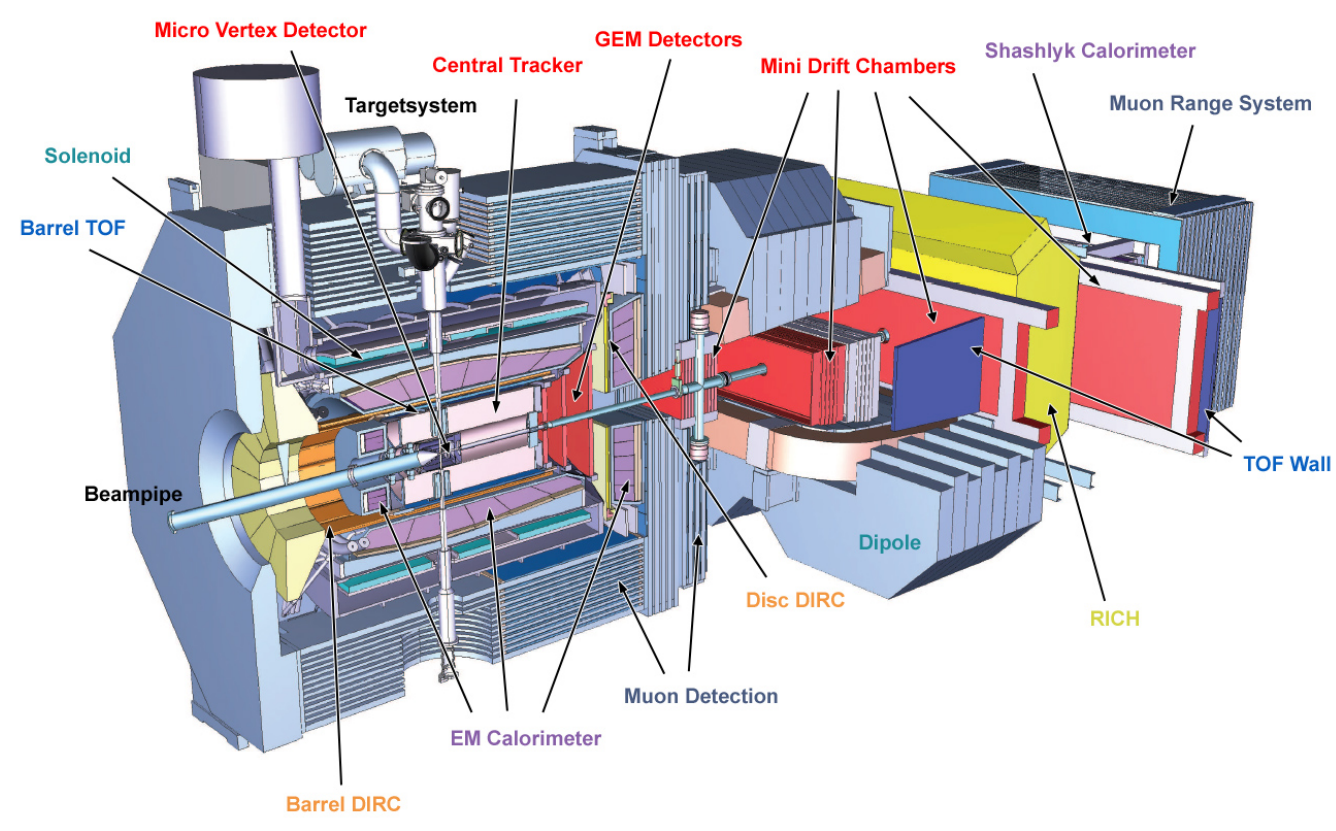

Figure 1: Layout of the $\bar{P} A N D A$ detector consisting of a target spectrometer, surrounding the interaction region, and a forward spectrometer to detect particles emitted in the forward region. The HESR antiproton beam enters the apparatus from the left side.

The $\bar{P}$ ANDA experiment will cover a rich and diversified hadron physics program, from charmonium spectroscopy to hypernuclear physics, with the capability to investigate also gluonic excitations and electromagnetic processes (more details in [1]). In order to satisfy the requirements of such a broad physics program, particle identification (PID) assumes a central role. In the barrel region of the $\bar{P}$ ANDA detector, a DIRC will take care of charged particle identification, with the aim to get a $3 \sigma$ separation of charged $\pi$ and $\mathrm{K}$ up to $3.5 \mathrm{GeV} / \mathrm{c}$ [2]. A DIRC is a 2D device providing a pattern of the projected Cherenkov light allowing to determine the particle species. The time information is required to suppress background and also apply chromatic corrections, depending on the time resolution. In the PANDA experiment Cherenkov imaging needs photomultipliers able to work in a strong magnetic field $(\sim 1-2 \mathrm{~T})$, together with high granularity and fast timing, therefore

\footnotetext{
${ }^{1}$ antiProton ANnihilations at DArmstadt
} 
MCPs have been chosen for the read-out of the DIRC. Considering the features behind the DIRC detector principles and MCP properties (further details in [3]), the development of dedicated FEE has to consider the following key points:

- single photon detection: on average 15-20 photoelectrons per track are expected and therefore the capability to detect small MCPs signal ( $\sim 2 \mathrm{mV})$ is essential to extract PID information from pattern recognition of Cherenkov images;

- about 15000 channels: the photodetector plane in the expansion volume requires a large number of channels besides a high pixel density to fulfill the imaging needs;

- good time resolution: due to the DIRC characteristics a resolution $<100 \mathrm{ps}$ is needed to apply chromatic corrections [4];

- rate per channel of 50-100 KHz: even if the number of photoelectrons per track is small, FEE needs to be fast in order to work with high rate of HESR.

The aim is to develop a versatile FEE able to meet the listed targets.

\section{The DIRC Prototype}



Figure 2: Schematic drawing of the DIRC prototype.

The development of dedicated FEE started around a basic and adaptable prototype of the DIRC detector. A synthetic fused silica bar $\left(35 \times 17 \times 800 \mathrm{~mm}^{3}\right.$, with $n \approx 1.47$ at $\lambda \approx 400 \mathrm{~nm}$ [5]) is used as radiator so that charged particles passing through with $\beta=v / c>1 / n$ emit Cherenkov photons at an angle $\Theta_{C}=\arccos (1 / n \beta)$ [3]. The bar works also as a light guide and Cherenkov photons confined in the bar are internally reflected. The absolute value of $\Theta_{C}$ is conserved due to the excellent surface polish. One of the two edges of the bar is optically coupled to a planoconvex lens, followed by an adjustable air-gap and an expansion volume $\left(800 \times 600 \times 300 \mathrm{~mm}^{3}\right)$ filled with oil (Marcol82, $n \approx 1.47$ ). An array of MCPs, 64 channels with an $8 \times 8$ matrix (Burle XP85012), is placed on the opposite side of the expansion volume which corresponds to the focal plane. After the MCPs follows the electronic read-out chain, composed of FEE boards, directly linked to the photomultipliers, and TRBv3 (see fig. 2). The acquisition system is managed by the new TRBv3, developed by GSI. The board consists of 4 general purpose Field-Programmable Gate Array (FPGA), in which 16-channel time-to-digital converters are implemented. High flexibility and high time resolution $\left(\sigma_{t} \sim 7 \mathrm{ps}\right.$ on a single channel) are important features of TRBv3 [6]. An 
alternative and simplified version of the prototype has been realized for the preliminary studies of the FEE. In this case the bar is directly attached to one MCP, followed by the same electronics chain.

\section{Front-End Electronics development}

The development of dedicated FEE has been done at the Institut für Kernphysik in Mainz. To meet the required targets, the understanding of MCP output is of crucial importance. The typical signal, which arises from a visible photon hitting the photodetector surface, has a rise time of $\sim 600 \mathrm{ps}$, a pulse width of about $2 \mathrm{~ns}$ and, as shown in [7], a single photon time resolution $\sigma_{t}<50 \mathrm{ps}$. Considering a typical gain of $6 \cdot 10^{5}$ the collected charge is $\sim 100 \mathrm{fC}$, which means a peak amplitude of few (2-5) $\mathrm{mV}$ using a typical $50 \Omega$ termination. Such low signals are at the limit for use with conventional discriminators, so a pre-amplification stage is needed. A modular FEE system, shown in fig. 3 , has been developed using the following boards:

- Preamplifier board: an ultra low-noise current feedback amplifier (THS3201 of Texas Instruments), with a bandwidth up to $1.8 \mathrm{GHz}$ and a gain $\sim 8$. A total of 16 channels is implemented (8 per layer);

- NINO ASIC board: an ultra low power discriminator with differential input and LVDS output. Two 8 channels NINO chips, produced by CERN, provide a total of 16 channels per board, each of them with adjustable discriminator threshold. High rates $(>10 \mathrm{MHz})$ are sustained with a front end time jitter <10ps [8].

The idea behind the FEE design is to obtain a discriminated signal whose Time over Threshold (ToT) is proportional to the charge of the original MCP signal. In fig. 3 is shown an example of a preamplified signal and the corresponding LVDS NINO output. The modular system will allow to investigate possible alternatives to ToT, such as charge-to-width or pulse height measurements, keeping the same preamplifier and replacing only the discriminator board.
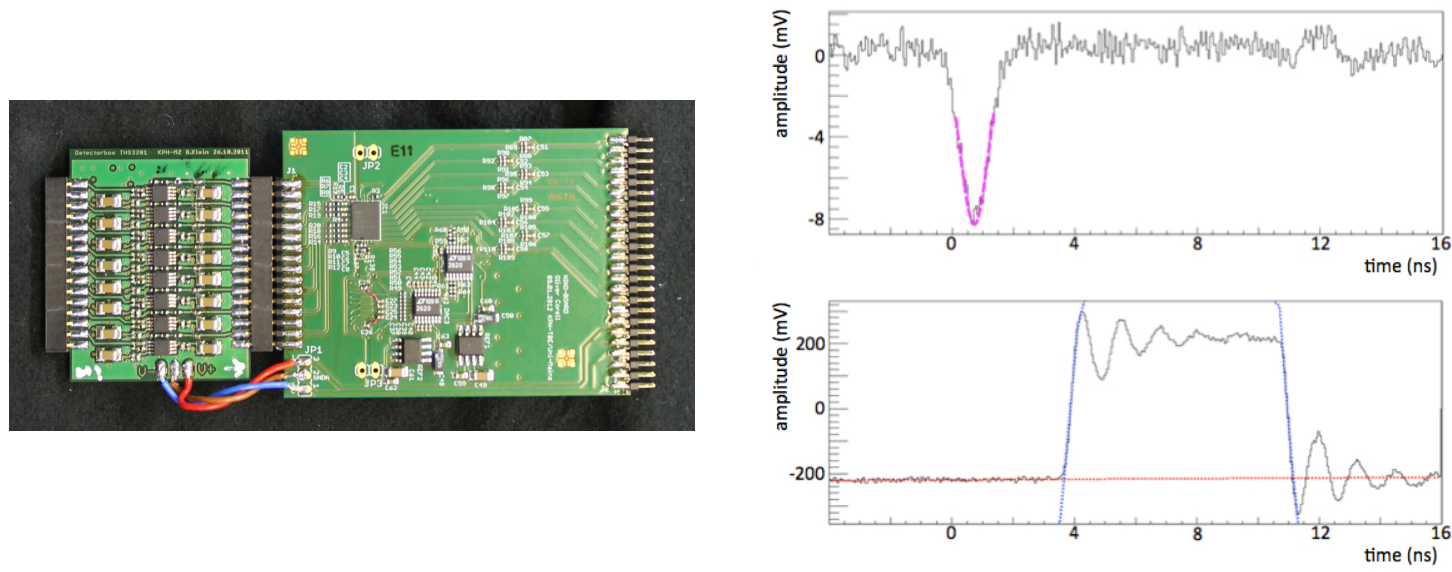

Figure 3: Left: FEE electronics. Left board is the Preamplifiers while right one is the NINO ASIC board. Top Right: example of preamplifier signal. Bottom Right: corresponding LVDS NINO output. 


\subsection{Time over Threshold Studies}

The aim of the FEE characterization is to establish if ToT could be used to correct the time walk of the original MCP signal. The study has been done considering a data set of Cherenkov photons from a preliminary in-beam test at Mainz, where a single MCP was connected directly to the fused silica bar. Measurements focused on a single MCP channel have been done with a fast oscilloscope (20 Gsamples/s and bandwidth of $6 \mathrm{GHz}$ ) so that the complete waveforms of preamplifier and NINO signals were available for offline analysis. In this way the time walk, $t_{\text {walk }}$, has been defined as the time distance between the leading edge of the preamplifier signal and the leading edge of the NINO (see fig. 3 , right). The width of the $t_{\text {walk }}$ distribution provides an estimate of the error introduced by the FEE. In the ideal case $t_{\text {walk }}$ should not depend on the signal amplitude $h_{\text {sig }}$ while, indeed, a clear correlation has been found (see fig. 4, left). A fit $t\left(h_{\text {sig }}\right)$ has been applied using a second and first order polynomial, respectively for low and high amplitudes. The ideal correction for $t_{\text {walk }}$ can be written as:

$$
t_{\text {corr }}=t_{\text {walk }}-t\left(h_{\text {sig }}\right)+\text { offset. }
$$

Since the NINO output is proportional to the ToT of the signal, the correction needs to consider the relation between ToT itself and amplitude, $h_{s i g}^{*}(T o T)$. As follows it is possible to evaluate the amplitude starting from the ToT, hence:

$$
t_{\text {corr }}=t_{\text {walk }}-t\left(h_{\text {sig }}^{*}(T o T)\right)+\text { offset. }
$$

The results show that, when 3.2 is applied, the standard deviation $\sigma$ of $t_{\text {walk }}$ distribution decreases from $0.22 \mathrm{~ns}$ to $0.16 \mathrm{~ns}$ (see fig. 4 , right). This means that ToT can be used to correct $t_{\text {walk }}$, leading to a relative improvement of $\sim 27 \%$. Similar results have been found using signals from MCP dark noise.

The contributions to the time walk need further investigations, e.g. for the NINO ASIC it is known that the time walk is correlated with ToT [8].
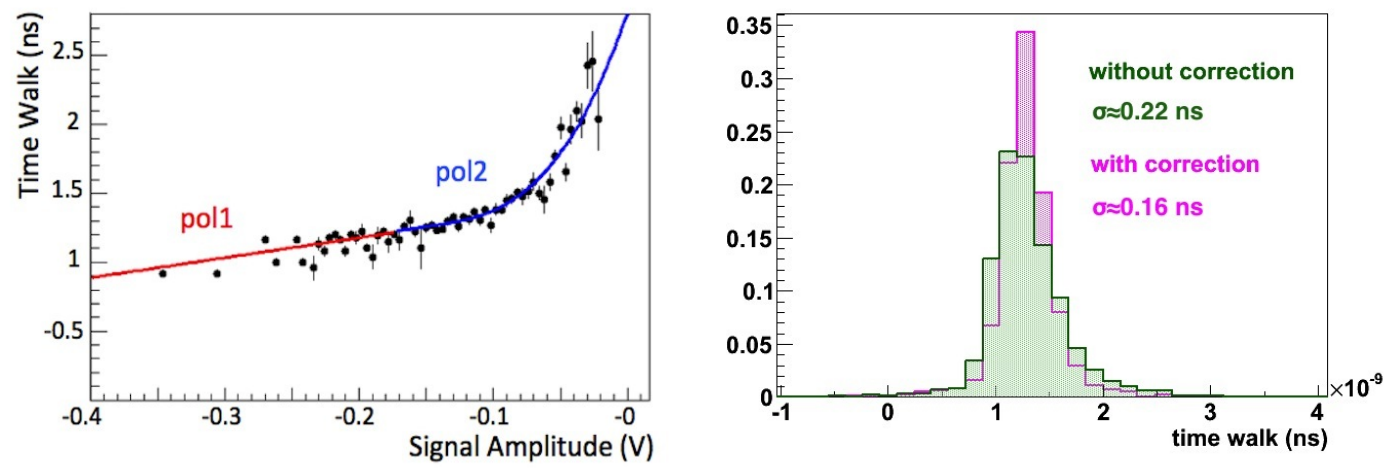

Figure 4: Left: Time walk as function of the signal amplitude (or pulse height). Top Right: in green time walk distributions without correction, in pink after correction. Both distributions are normalized to 1. 


\section{In-beam test at MAMI-X1}

An in-beam test of the complete setup (see section 2) has been performed at the X1 line of the MAMI accelerator in Mainz. The continuos wave electron beam has an energy of $855 \mathrm{MeV}$, a diameter of $1 \mathrm{~mm}$ and a maximum divergence of $0.1 \mathrm{mrad}$, features that make this facility ideal for high precision test of detector prototypes like the DIRC. The aim of the test was to prove the full electronics chain under real conditions and the ability to detect Cherenkov patterns.

A vertical array of 3 MCPs has been used to detect the Cherenkov images in the focal plane of the expansion volume. The system has been completed by 3 TRB boards ( 1 per MCP) in which 128 TDC channels have measured the leading and trailing edges of the $64 \mathrm{MCP}$ channels. The acquisition rate has been fixed to $100 \mathrm{kHz}$. In fig. 5 the results for a run $(\sim 600.000$ events $)$ with an incidence angle of $30^{\circ}$ with respect to the bar are shown; the number of hits per channel is given by the color scale (red high number, blue low one). A Monte Carlo simulation (black dots) with 1000 events has been used to verify the agreement with the position of the hit pattern on the MCPs. White areas in the plots are due to electronics issues while the interrupted pattern of the signal is probably due to incorrect thresholds settings of the FEE cards. The result permits to identify the found structure as Cherenkov ring. Further studies, varying the impact angle between bar and beam, have corroborated that the image was moving in agreement with expectation.
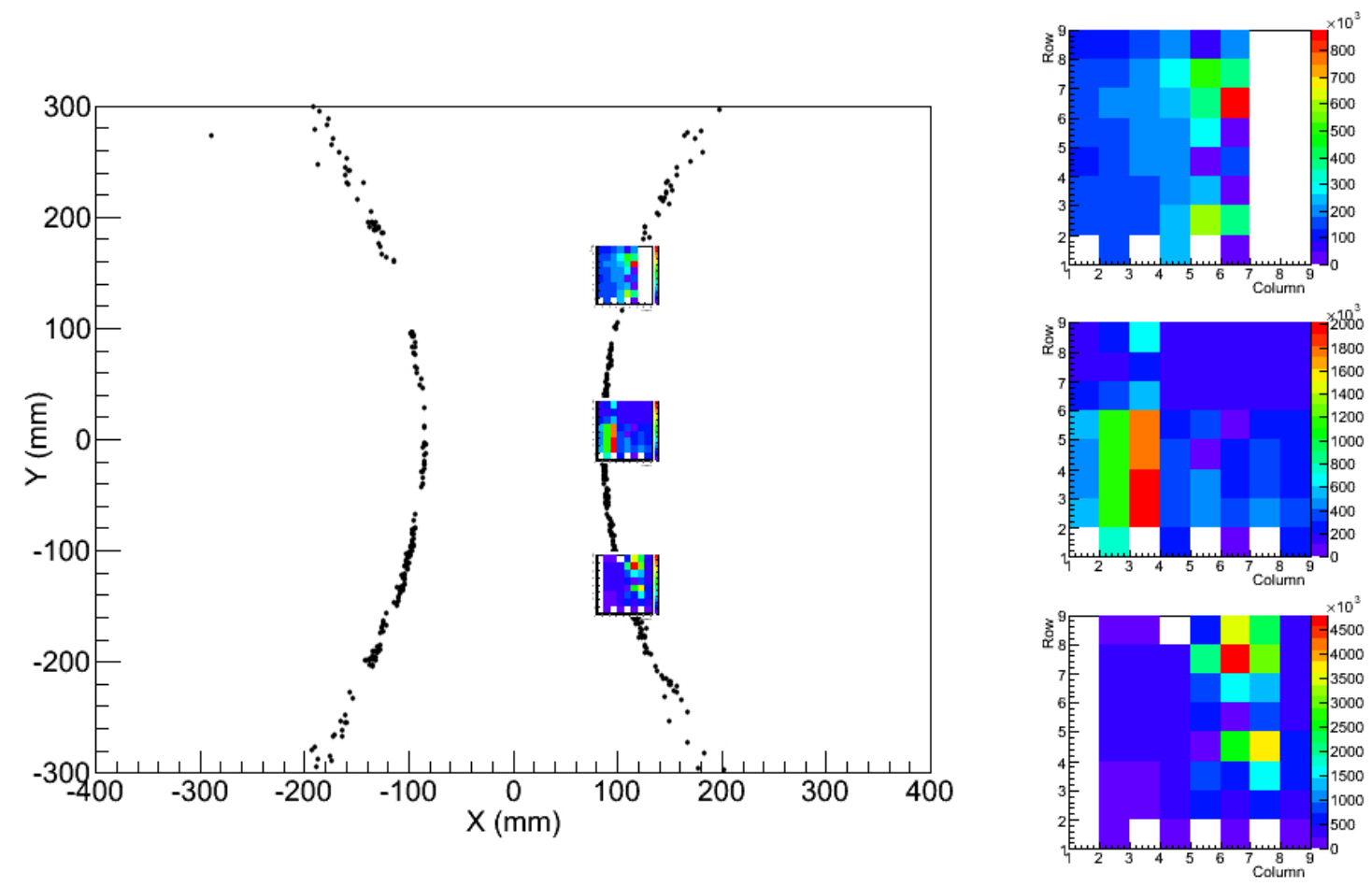

Figure 5: Left: Result of in-beam test, black point are Monte Carlo simulations while the 2D histograms are the hit patterns on the MCPs over a complete run. Right: Zoom in of the three MCPs histograms. The number of hits per channel is given by the color scale. 


\section{Conclusions and Outlook}

The development of versatile and adaptable FEE for the PANDA DIRC has been successfully addressed. A system composed of a preamplifier and a discriminator board has been realized to meet the detector requirements and the MCP features. The implemented ToT logic has proved to decrease the time walk by $\sim 27 \%$. The full chain has been studied at the Mainzer microtron MAMI with an in-beam test, which established the ability to detect Cherenkov patterns, working with a rate of $100 \mathrm{KHz}$ as required for $\overline{\mathrm{P}} \mathrm{ANDA}$.

Timing studies showed a resolution of $160 \mathrm{ps}$ which is not meeting the target of $100 \mathrm{ps, \text {therefore, }}$ a laser setup is foreseen to perform a full characterization of the FEE. In the second generation, other solutions for the discriminator stage will be tested to improve the time walk correction. In particular designs with charge directly measured on board are currently under study. Alternative FEE concepts with FPGAs as discriminators are also under investigations.

Further in-beam tests at MAMI are foreseen to continue and complete the electronics development.

\section{References}

[1] PANDA collaboration, Physics Performance Report for $\bar{P} A N D A$ : Strong Interaction Studies with Antiprotons, ArXiv:0903.3905, (2009)

[2] C. Schwarz et al., Particle identification for the $\bar{P} A N D A$ detector, Nuclear Intruments and Methods in Physics Research A639 (2011) 169-172.

[3] B. N. Ratcliff, Imaging rings in Ring Imaging Cherenkov counters, Nuclear Intruments and Methods in Physics Research A502 (2003) 211-221.

[4] J. Schwiening et al., The barrel DIRC detector for the $\bar{P} A N D A$ experiment at FAIR, Nuclear Intruments and Methods in Physics Research A639 (2011) 315-318.

[5] Heraeus Quartz Glass for Optics, Data and Properties, HQS-MO_01.0/E/04.2013

[6] C. Ugur et al., Implementation of a High Resolution Time-to-Digital Converter in a Field Programmable Gate Array, in proceedings of 50th International Winter Meeting on Nuclear Physics, PoS (Bormio2012) 015.

[7] A. Lehmann et al., Systematic studies of micro-channel plate PMTs, Nuclear Intruments and Methods in Physics Research A639 (2011) 144-147.

[8] F. Anghinolfi et al., NINO, an ultra-fast, low-power, front-end amplifier discriminator for the Time-Of-Flight detector in ALICE experiment, Nuclear Instruments and Methods in Physics Research A533 (2004) 183-187. 